\title{
USE OF GLYCOGEN PHOSPHORYLASE BB MEASUREMENT WITH POCT IN THE DIAGNOSIS OF ACUTE CORONARY SYNDROMES. A COMPARISON WITH THE ELISA METHOD.
}

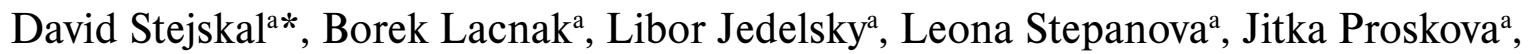 \\ Pavlina Solichova $^{a}$, Lenka Kadalova ${ }^{\mathrm{a}}$, Marie Janosova ${ }^{\mathrm{a}}$, Petra Seitlova ${ }^{\mathrm{a}}$, Michal Karpisek ${ }^{\mathrm{b}}$, \\ Ludek Sprongl ${ }^{\mathrm{c}}$
}

\author{
a Department of Laboratory Medicine and Department of Internal Medicine, Sternberk Hospital, Czech Republic \\ ${ }^{b}$ Institute of Human Pharmacology and Toxicology, Faculty of Pharmaceutical Sciences, Veterinary and Pharmaceutical \\ University Brno, Czech Republic \\ c Central Laboratory, Sumperk Hospital, Czech Republic \\ e-mail:david.stejskal@nemstbk.cz
}

Received: August 21, 2007; Accepted (with revisions): November 10, 2007

Key words: Acute coronary syndrome/Myocardial ischemia/Non-STEMI/Coronary by-pass/Laboratory markers of myocardial ischemia/CAD/GPBB/POCT/ELISA

Background: Glycogen Phosphorylase BB (GPBB) is considered an early and specific marker of myocardial necrosis and ischemia. A POCT kit GPBB for diagnostic use has recently been approved.

Aim: an evaluation of the correspondence of qualitative POCT GBPP measurements with ELISA test results.

Material and methodology: 20 individuals with non-ST elevation myocardial infarction (non-STEMI) and 20 probands without acute coronary syndrome (ACS) were tested. GPBB (POCT, ELISA) in venous plasma (lithium-heparin) was assayed in all probands.

Results: individuals with non-STEMI had significantly higher GPBB ELISA values (32.3 vs. 6.1 $\mu \mathrm{g} / 1 ; \mathrm{p}<0.01)$. GPBB sensitivity and specificity for non-STEMI presence 6 hours after chest pain generation were $100 \%$. No proband was classified in a different subgroup with POCT of GPBB (positive/negative). GPBB POCT correlate with a nonSTEMI diagnosis $\left(\chi^{2} 36.1 ; \mathrm{p}<0.01\right)$.

Conclusion: GPBB POCT measurement is comparable with ELISA test results. GPBB analysis could expand the diagnostic palette in the first hours after the onset of acute coronary syndrome.

\section{INTRODUCTION}

Routinely used laboratory markers for the presence of myocardial necrosis are similar in that they diagnose myocardial necrosis relatively late (for example, cardiac troponins) or they are characterized by low specificity (for example, myoglobin or albumin binding cobalt-ACB)(ref. ${ }^{1,8,9}$ ). Even more problematic is the situation in diagnosing the presence of myocardial ischemia without necrosis when regularly-used markers are non-specific and the diagnostician can be burdened by a relatively large error rate (for example, ACB) ${ }^{1}$.

From this perspective Glycogen Phosphorylase Isoenzyme BB (GPBB) appears to be a promising, early specific marker of myocardial necrosis (early release after the first two hours). In addition, information has recently appeared regarding its diagnostic use in the assessment of reversible myocardial ischemia ${ }^{1-3}$. A few years ago the first diagnostic kits for measuring GPBB (ELISA, Diagenics) appeared on the market ${ }^{7}$; in our own group of individuals suspected of having acute coronary syndrome we verified the great diagnostic efficiency of this marker in the early stages of myocardial necrosis and ischemia ${ }^{11}$. As the manufacturer of the ELISA kit also offers the possibility of determining GPBB through POCT (point-of-care-test), we posited the question in our work whether GPBB results obtained by the POCT method are comparable with the results obtained from the ELISA test.

\section{METHOD}

Forty individuals hospitalized in the Department of Internal Medicine at Šternberk Hospital for suspected acute coronary syndrome (ACS) were chosen for testing. Twenty of these patients were diagnosed as positive (acute myocardial infarction without ST segment elevation) and twenty were diagnosed as negative.

The final diagnosis of non-STEMI (non-ST elevation myocardial infarction) / non-coronary difficulties was made on the basis of ESC/AAC criteria (medical history, clinical findings, EKG, troponins, echocardiography); only individuals with a positive coronarographic finding (i.e. these were not consecutive patients) were chosen for 
Table 1. Distribution of GPBB ELISA in individuals by ACS presence

\begin{tabular}{|c|c|c|c|c|c|c|c|c|c|c|}
\hline \multirow[b]{2}{*}{ Parameter } & \multicolumn{4}{|c|}{ Individuals with non-STEMI $(\mathrm{n}=20)$} & \multicolumn{4}{|c|}{ Individuals without ACS $(\mathrm{n}=20)$} & \multirow{2}{*}{ F ratio } & \multirow{2}{*}{$\mathrm{P}$} \\
\hline & $\mathrm{X}$ & Median & SD & Normality & $\mathrm{X}$ & Median & SD & Normality & & \\
\hline GPBB & 32.3 & 32.6 & 14.7 & Yes & 6.1 & 6.2 & 1.4 & Yes & 63.6 & $<0.01$ \\
\hline
\end{tabular}

the group of patients with ACS evaluated in our study. The patients that we put in the non-coronary pain category had negative troponin, negative echocardiographic and electrocardiographic findings; the source of pain was subsequently determined for these patients (by exercise test with bicycle ergometry, and if necessary SPECT, neurological examination, pneumological examination, rehabilitation examination, and similar methods) and was explained as non-coronary (for example thoracalgia musculoskeletal etiology, vegetative dystonia, pleural syndrome, and others).

Venous blood was drawn from all individuals in the framework of this study for the purpose of determining GPBB. In all patients, the laboratory examination of GPBB in venous blood was made immediately after admission (6 hours of chest pain in patient's history at the most; mean period of chest pain since the beginning was 3.2 hours). The blood was placed in tubes with lithiumheparin (Sarstedt, catalogue no. 04.1936); within thirty minutes from the time the blood was drawn separation was performed on the samples in a cooled centrifuge $\left(3,000 \mathrm{~g}, 10 \mathrm{~min}\right.$.) to acquire plasma frozen to $-80{ }^{\circ} \mathrm{C}$. ELISA and POCT tests were conducted simultaneously within 90 days of the freezing. ELISA kit developer declared stability of GPBB in noted temperature for the duration of storage.

The data were processed using the Medcalc programme (Belgium). The Komolgorov-Smirnov test was used to evaluate the normality of the GPBB data from the ELISA test. Continuous values were expressed as the means \pm standard deviations and median. ANOVA was used to evaluate GPBB-ELISA for the presence of ACS and ROC analysis was used to evaluate diagnostic efficiency. A value $p<0.05$ was considered to be statistically significant.

\section{RESULTS}

Twenty individuals were determined to be non-STEMI, while the remaining twenty difficulties were determined to be non-coronary.

Non-STEMI patients had significantly higher GPBBELISA values (Tab. 1). The sensitivity and specificity of GPBB for the presence of non-STEMI 6 hours after chest pain generation amounted to $100 \%$ with cut-off value of $8.5 \mu \mathrm{g} / 1$ (Fig. 1). When the manufacturer's recommended cut-off value was used $(10 \mu \mathrm{g} / \mathrm{l})$ the sensitivity and specificity results remained the same.

A comparison of the ELISA results using the manufacturer's recommended cut-off value showed that all 40

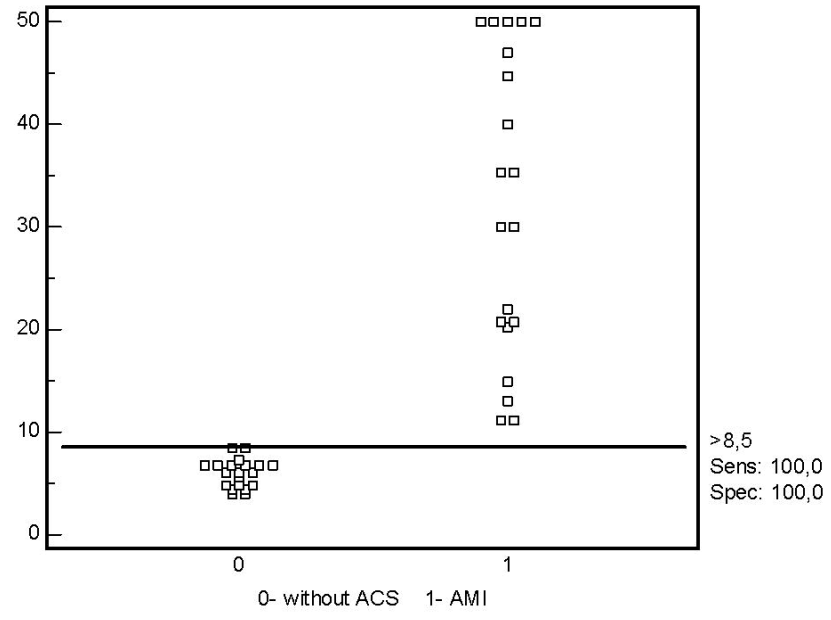

Fig. 1. Interactive plot diagram for GPBB ELISA $(\mu \mathrm{g} / \mathrm{l})$ and non-STEMI presence ( 6 hours after the admission, AMI). AUC 1.0 (95 \% CI 0.91 - 1.0).

examined individuals ( $100 \%$ ) were correctly identified as positive (20 patients) and negative (20 patients). None of the patients was diagnosed differently (positive/negative) the POCT method and during the interpretation of ELISA results. A table with four fields shows that the GPBB POCT results correspond with the non-STEMI diagnosis $(36.1 ; \mathrm{p}<0.01)$.

\section{DISCUSSION}

Glycogen Phosphorylase (Glycogen Phosphorylase; -1.4-D-glucan: orthophosphate D-glucosyltransferase; GPBB; EC 2.4.1.1) is a glycolytic enzyme that plays an essential role in the regulation of carbohydrate metabolism. It catalyzes the first step of glycogenolysis, in which glycogen is converted to glucose 1-phosphate (mobilizes glycogen, which is connected to the delivery of glucose). These conditions appear during ischemia and hypoglycaemia. GPBB is connected with glycogen in a macromolecular complex that is structurally bound to the sarcoplasmic reticulum (the sarcoplasmic reticulum glycogenolysis complex). The degree of GPBB association with this complex depends above all on the metabolic state of the individual. During hypoxia, GPBB is released from the bond a socalled soluble form of GPBB predominates and escapes the cell through diffusion. The early release of GPBB into the blood is a common result of the combination of escalated glycogenolysis and increased permeability of cell 
membranes which is typical for myocardial ischemia and necrosis ${ }^{1}$.

Under physiological conditions, Glycogen Phosphorylase is a dimer (monomer has $\mathrm{Mr} 97 \mathrm{kDa}$ ), which is composed of two identical subunits (homodimer). Three enzymes are commonly found in human tissue - GPLL (liver isoform), GPMM (muscle isoform), and GPBB (brain isoform.) These isoenzymes are encoded by different genes and differ in both structure and function. The $\mathrm{BB}$ isoenzyme is found in brain and heart muscle and in small amounts in striated muscles in other tissue as well (in leukocytes, spleen, kidneys, bladder, testes, liver, digestive tract, and aorta $)^{1}$.

Following the results of clinical studies, GPBB can be regarded as a highly sensitive marker that is released 1 to 4 hours after the onset of acute myocardial infarction (AMI), while $100 \%$ of patients showed elevated GPBB after 4 to 5 hours $^{1-3,5}$. GPBB is also a sensitive marker for the detection of perioperational myocardial ischemia and it is possible that it could also be used as a marker of early reperfusion. Values normalize 1 to 2 days following the onset of $\mathrm{AMI}^{1,4,5,8}$. False positives for increased GPBB are found in patients with trauma or significant damage to the central nervous system? .

It is highly interesting that GPBB also increases in patients with acute coronary syndrome without of myocardial ischemia; the determination of GPBB has a high prognostic value for these individuals ${ }^{2,3}$. It is likely that GPBB could, unlike myoglobin or troponins, identify myocardial ischemia, since only GPBB increases above the upper reference limit during of these conditions ${ }^{3,5}$.

Commercial kits for determining GPBB levels were not available a few years ago (only in-house ELISA methods existed $)^{2,6,10}$. Diagnostic kits have only recently appeared on the market (approved by the FDA and labelled CE) based on ELISA and for the POCT regime. Principle of the both tests is similar ${ }^{7}$. GPBB-ELISA (Enzyme-Linked Immunoadsorbent Assay) is a blood test for the early detection of myocardial infarction. It works on an (sandwich assay, two antibodies) enzyme basis ${ }^{7}$. GPBB-POCT (The Point Of Care Test) is the successor of the ELISA test and an important supplement for the emergency ambulances. It is a quick and qualitative test for making the first decisions. POCT is a stripe test (lateral flow), which also functions on the GPBB basis. Its various layers are plated with small traces of monoclonal catch-antibody of GPBB. Another antibody, responsible for the colouring, is applied on the other side. Once the blood sample runs on the absorbent strap on the antibody track, GPBB is caught by the antibodies and made visible with the help of the antibody colouring. $50 \mu \mathrm{l}$ are enough to gain a solid result. The time for testing only takes about 15 minutes ${ }^{7}$. GPBB-POCT test can be performed using full blood (test tube with lithium-heparin), plasma (lithium-heparin), or serum. In the event that GPBB is present in the sample in a concentration exceeding $10 \mu \mathrm{g} / 1$ a coloured line will appear (samples are evaluated as positive). An additional coloured line always appears to control the accuracy of the test. The manufacturer holds a patent on the production and clinical use of this test ${ }^{7}$.
Our results confirmed the absolute correspondence between the results of the ELISA test and qualitative GPBB test in the POCT regime. We are aware of the qualifying fact that our test involved individuals with acute myocardial infarction (non-STEMI) 6 hours following onset and their comparison with individuals without ACS, when with respect to time factors the determination of heart troponins is optimal for the diagnosis. Nevertheless, our goal in this work was not to confirm the exceptionality of GPBB as a fast marker (this was previously conducted) ${ }^{11}$, but to confirm the correspondence of GPBB results measured by ELISA and POCT.

It is possible to conclude that GPBB from POCT provides, at the recommended cut-off value results that are comparable with values determined by the ELISA test. It is reasonable to assume that the GPBB POCT test can expand the laboratory diagnostic palette in the first hours following acute coronary syndrome. The verification of the singular early diagnostic efficiency of this test could even be interesting for manufacturers of automatic immuno-analytical systems.

\section{REFERENCES}

1. Apple FS, Wu AHB, Mair J, Ravkilde J, Panteghini M, Tate J, Pagani F, Christenson RH, Mockel M, Danne O, Jaffe AS. Future Biomarkers for Detection of Ischemia and Risk Stratification in Acute Coronary Syndrome. Clin. Chem 2005; 51:810-824.

2. Mair J, Puschendorf B, Amidy J, Lechleitner P, Dienstl F, Noll F, Krause EG, Rabitzsch G. Early release of glycogen phosphorylase in patients with unstable angina and transient ST-T alterations. British Heart Journal 1994; 72:125-127.

3. Rabitzsch G, Mair J, Lechleitner P, Noll F, Hofmann U, Krause EG, Dienstl F, Puschendorf B. Immunoenzymometric assay of human glycogen phosphorylase isoenzyme BB in diagnosis of ischemic myocardial injury. Clin. Chem 1995; 41:966-978.

4. Mair P, Mair J, Krause EG, Balogh D, Puschendorf B, Rabitzsch G. Glycogen phosphorylase isoenzyme BB mass release after coronary artery bypass grafting. Eur J Clin Chem Clin Biochem 1994; 32:543-547.

5. Peetz D, Post F, Schinzel H, Schweigert R, Schollmayer C, Steinbach K, Dati F, Noll F, Lackner KJ. Glycogen phosphorylase BB in acute coronary syndromes. Clin Chem Lab Med. 2005; 43:1351-1358.

6. Hofmann U, Rabitzsch G, Loster K, Handschack W, Noll F, Krause EG. Immunenzymometric assay for the heart specific glycogen phosphorylase BB in human serum using monoclonal antibodies. Biomed Biochim Acta 1989; 48:132-136.

7. Diagenics [online]. Dostupné z WWW < http://www.diagenics. de

8. Kemp M, Donova J, Higham H, Hooper J. Biochemical markers of myocardial injury. British Journal of Anaesthesia 2004; 93:6373.

9. Wu AHB, Apple FS, Gibler WB, Jesse RL, Warshaw MM, Valdes R. National Academy of Clinical Biochemistry Standards of Laboratory Practice: Recommendations for the Use of Cardiac Markers in Coronary Artery Diseases. Clin Chem 1999; 45:11041121.

10. Mair J. Glycogen phosphorylase isoenzyme BB to diagnose ischaemic myocardial damage. Clin Chim Acta 1998; 272:79-86.

11. Proskova J, Stejskal D, Lacnak B, Vaclavik J, Solichova P, Karpisek M, Kaminek M. Glycogenphosphorylase BB in the early diagnosis of ACS. Clin Chem Lab Med 2007; S1:W288. 